\title{
Using Mandatory Data Collection on Multiresistant Bacteria for Internal Surveillance in a Hospital
}

\author{
U. Sagel', R. T. Mikolajzzyk², A. Krämer ${ }^{2}$ \\ IInstitute of Microbiology and Infectious Diseases Epidemiology of Gilead/Mara Hospitals, \\ Bielefeld, Germany \\ ${ }^{2}$ Department of Public Health Medicine, School of Public Health, University of Bielefeld, Germany
}

\section{Introduction}

Objectives: Multiresistant pathogens cause major clinical problems and considerably increase treatment costs. Since 2001 the Protection Against Infection Act (PIA) obligates hospitals in Germany to the documentation of multiresistant bacteria. We analyzed the use of these data for routine internal surveillance.

Methods: We used standard data collected for the mandatory documentation and studied consecutive diagnoses of Methicillin-resistant Staphylococcus aureus (MRSA) in a 893-bed tertiary level hospital in North Rhine-Westphalia in Germany. Based on the Poisson distribution for the cumulative yearly incidence of MRSA, we defined a threshold level for an outbreak. Results: During a 12-month time period 80 patients were diagnosed with MRSA. The time structure and spatial distribution of different MRSA phenotypes (defined through specific antibiotic resistance patterns) were consistent with the within-hospital transmission. In the two preceding time periods of 12 months each, 15 respectively 8 patients with MRSA were found. The defined alert threshold level for cumulative yearly incidence was crossed in the beginnings of the outbreak. Conclusion: Monitoring the mandatory data collected on multiresistant bacteria allows the early detection of accumulations suspect for the within-hospital transmission. This knowledge can be used for a fast reaction and breaking off the transmission chains.

\section{Keywords}

Nosocomial infection, multiresistant pathogens, surveillance

Methods Inf Med 2004; 43: 483-5
Nosocomial infections are an increasing challenge for infectious diseases epidemiology in industrialized countries. Multiresistant pathogens cause major clinical problems and lead to higher treatment costs [1]. In 2001, a new regulation Protection Against Infection Act (PIA) was introduced with the purpose of improving the monitoring of multiresistant pathogens. According to $\$ 23$ of this law, the responsible German authority (Robert-Koch-Institute, Berlin) defined 12 bacterial pathogens and one fungal pathogen with resistance towards from one to six antibiotics (respectively groups of antibiotics) to be of special concern and to be mandatorily recorded by all German hospitals [2]. This definition does not distinguish between multiresistant pathogens and those that are resistant against only one broad-spectrum antibiotic. We studied the suitability of these mandatory data for the internal surveillance in the case of an outbreak with methicillin-resistant Staphylococcus aureus (MRSA).

\section{Methods}

During the time from June 2002 to July 2003, an increased number of MRSA cases was observed in a 893-bed tertiary level hospital in the state North Rhine-Westphalia, Germany. We studied the mandatory data on multiresistant bacteria and analyzed the occurrence of same species with same resistance patterns in time and spatial distribution over the different wards. When cases appeared connected, transmission chains were suspected.

We defined a threshold level for an outbreak when the number of observed MRSA cases is unlikely to result from admission of patients already colonized with MRSA. Given the large number of patients treated in a year in the hospital and the low number of MRSA cases observed in the previous years, when there was no evidence for an outbreak, the probability for a specific number of MRSA cases can be derived from the Poisson distribution. The mean of the cumulative incidence observed in two previous time periods of 12 months is used as the mean of the Poisson distribution and percentiles are calculated. The threshold is defined as the probability of $<5 \%$ in support of no transmission (i.e. when only patients already colonized at admission contribute to the cumulative incidence). When a new patient is diagnosed with MRSA, the number of MRSA in 12 months ending on the day of the new diagnosis is calculated and compared with the threshold.

\section{Results}

Between July 2002 and June 200380 patients with MRSA were observed in the hospital under study. In the two preceding 12 -month periods the respective numbers were 15 and 8 patients. Fifty-seven of the 80 patients had a specific pattern of resistance (phenotype). Isolates from four of these patients proved to be genotypically identical and were classified as a sub-clone of "southern German MRSA strain" (Witte W, 


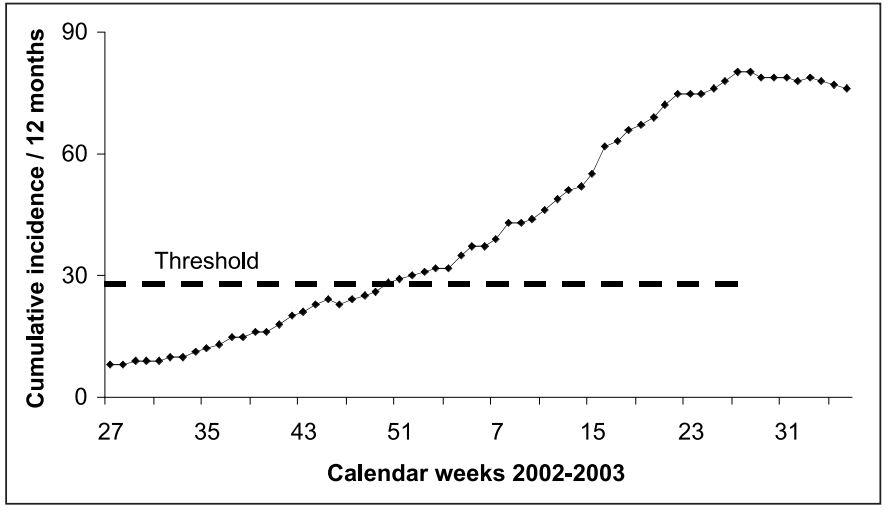

Fig. 1

Cumulative incidence of MRSA cases per 12 months and alert threshold for an outbreak

national reference laboratory for Staphylococcus, Wernigerode, Germany).

Most of the cases ( 45 of the 57 cases with the specific phenotype) were recorded on a 14-bed surgical intensive care unit (ICU). In the preceding time period of 12 months only two cases of MRSA were observed on the ICU. To rule out that an improved detection of multiresistant pathogens was the cause for an increased incidence of MRSA on ICU, we compared the number of bacterial specimen collected over the period of the outbreak and during the preceding year. Except for a slight increase on the peak of the outbreak the number of specimen remained stable. Similarly the increase in multiresistant pathogens could not be caused by an increase in the number of patients staying on the ICU, because these parameters remained stable. We are not aware of any changes in diagnostic standards or regime, which could cause an increased detection, either.

Most of the patients with MRSA observed on other wards could have been infected in connection with the cluster on ICU. Some of these patients were for some time patients on the ICU. Others had contacts with patients from the ICU.

In addition, analysis of all multiresistant pathogens according to PIA has shown that 55 patients have been involved in clusters of bacteria with specific resistance patterns other than MRSA between July 2002 and June 2003. There were 23 cases with a multiresistant Acinetobacter baumannii (sensibility only towards carbapenems). Thirty-two patients were involved in clusters of other different bacteria that never contained more than four cases. Our further analysis focuses only on MRSA.

Besides the two preceding years for which we could obtain numerical information, we knew from personal information that the number of MRSA in previous years was relatively low. Accounting for some underreporting we assumed the usual number of MRSA cases to be at 20 in 12 months, this resulted in an alert threshold level at 28 patients in a year. The evolution of the outbreak in terms of cumulative incidence is displayed in Figure 1. The threshold outbreak developed quite slowly up to a level was crossed at the end of 2002. The

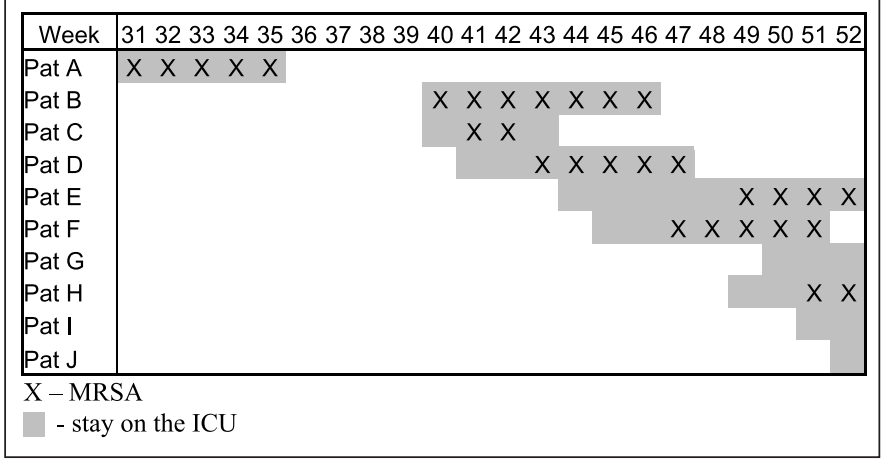

\section{Fig. 2}

Follow-up of the transmission chains of specific phenotype of MRSA on the ICU peak in April 2003, until it finally forced the hospital to take some vigorous action: In the beginning of May the ICU was closed temporarily and all patients with MRSA were discharged to other wards. Tracking the possible routes of infection we almost regularly found timely and spatial connections between the involved patients indicating possible chains of transmission. An excerpt of the analysis of these chains focusing on the ICU for the most prevalent phenotype of MRSA in the first weeks of the outbreak is shown in Figure 2. The spread of this phenotype started in the end of 2002, which is consistent with the observation from the threshold level.

\section{Discussion}

There are several limitations of our study. First, we did not obtain information on genotypes of the phenotypically identical bacteria except for four patients. Genotyping is costly and not routinely performed outside of university hospitals. We limited ourselves to the readily available mandatory data, which have the strong advantage that they exist for all hospitals adhering to the PIA. Resistance pattern phenotyping is regarded to be a method of good specificity and poor sensitivity to distinguish different bacterial clones [3, 4].

In epidemiology of German MRSA, data from 2002 indicate that prevalent genotypically determined clones mainly display different resistance patterns $[5,6]$. We assumed resistance pattern phenotyping not to be much less sensitive compared to genotyping in this special epidemiologic situation. More problematic is the fact that only few clones of MRSA spread in Germany (about 85\% belonging to four clones [5]) and quantitative data on their regional prevalence are not available. But these data are needed to determine the discriminatory sensitivity of genotyping with regards to determination of within-hospital transmission [7].

We used the Poisson distribution to define the threshold level for an outbreak. This is associated with two necessary decisions, first with the choice of baseline yearly inci- 
dence, which is regarded as normal, second with the choice of probability threshold for abnormal development. The baseline incidence should include only cases prevalent at admission to fulfil the requirement of statistical independency. The baseline incidence can be derived from observation of periods, where there is no evidence of transmission. Since the baseline incidence simply reflects the prevalence of MRSA at admission, screening of all patients at admission would provide a direct estimation. However, screening at admission is not performed in most hospitals in Germany. A more sophisticated way to obtain estimates of MRSA prevalence at admission can be based on mathematical modelling $[8,9]$. This would be of special value in settings with a higher endemic level of MRSA, where simple observation cannot distinguish between transmission and accumulation of cases colonized at admission.

The probability threshold for abnormal development (i.e. outbreak) has to be based on clinical judgement and balance the specificity and sensitivity of the method. Different approaches have been proposed for identification of abnormal patterns in occurrence of multiresistant pathogens based either on rather sophisticated techniques [10 - 13] or on comparison with other hospitals in the same region [14]. The advantage of our method is its simple use in routine settings based only on the mandatory data collected according to the PIA. It can be also used in regions with only few hospitals, where a comparison between different hospitals does not have statistical meaning. Although we did not present tables for the clinical use, the threshold levels adapted for a specific hospital can be simply developed.

A frequently discussed problem is the issue of underreporting of multiresistant bacteria. Since we observed no change in the number of specimen sent to the laboratory over a long period preceding the observed outbreak, there seems to be neither substantial over-detection nor under-detection in this case, at least on the level of the proxy. Nevertheless, in some cases the use of the same number of specimen could be shifted from patients with low probability of colonisation to patients with higher probability of infection. Such processes could particularly take place when the vigilance against MRSA is increased as during an outbreak. However this seems to have minor effects, especially on wards where the diagnostic procedures are highly standardized as on ICUs. In the particular case, microbiological monitoring was routinely performed twice a week (tracheal secrete and urine sampling). For clinical purposes tracheal secrete has the disadvantage of easy contamination by oropharyngeal flora, but it favors the detection of MRSA, which normally colonizes nose and pharynx.

We did not distinguish between infection and colonization with multiresistant bacteria, because both are of importance for the transmission dynamic.

We think that monitoring and systematical analysis of the mandatory data on multiresistant pathogens can be useful for internal surveillance on the hospital level. An alert threshold can be defined based on observations from previous time periods. Compared to other surveillance programs on multiresistant bacteria, our approach aims to be easily applicable in almost all German hospitals by using mandatory routine data from microbiological laboratories and a relatively simple epidemiological procedure. Observations from other outbreaks are needed to confirm our results.

\section{References}

1. Vincent JL. Nosocomial infections in adult intensive-care units. Lancet 2003; 361: 2068-77.

2. Anonymous. Surveillance of nosocomial infections and recording of pathogens with specific resistances and multiresistances [German]. Bundesgesundheitsbl - Gesundheitsforsch - Gesundheitsschutz 2000; 43: 887-90.

3. Pfaller MA, Acar J, Jones RN, Verhoef J, Turnidge J, Sador HS. Integration of molecular characterization of microorganisms in a global antimicrobial resistance surveillance program. Clin Infect Dis 2001; 32: S156.67.

4. Tenover FC, Arbeit RD, Goering RV, editors. How to Select and Interpret Molecular Strain Typing Methods for Epidemiological Studies of Bacterial Infections: A Review for Healthcare Epidemiologists. Infect Control Hosp Epidemiol 1997; 18: 426-39.

5. Anonymous. Staphylococcal infections in Germany in 2002 [German]. Epidemiol Bull 2003; 35: 277-80.

6. Witte W, Braulke Ch, Heuck D. MRSA-situation in Germany: Appearance, distribution and changes in virulent epidemic strains of MRSA with different resistance phenotypes [German]. Hyg Med 2000; 25 (9): 347-50.

7. Grundmann H, Hori S, Tanner G. Determining Confidence Intervals when Measuring Genetic Diversity and the Discriminatory Abilities of Typing Methods for Microorganisms. J Clin Microbiol 2001; 39: 4190-2.

8. Austin DJ, Bonten MJM, Weinstein RA, Slaughter S, Anderson R. Vancomycin-resistant enterococci in intensive-care hospital setting: Transmission dynamics, persistence, and the impact of infection control programs. Proc Natl. Acad Sci 1999; 96: 6908-13.

9. Pelupessy I, Bonten MJM, Diekmann O. How to assess the relative importance of different colonization routes of pathogens within hospital settings. Proc Natl Acad Sci 2002; 99: 5601-5.

10. Brown SM, Benneyan JC, Theobald DA, Sands K, Hahn MT, Potter-Bynoe GA, Stelling JM, O'Brien TF, Goldmann DA. Binary Cumulative Sums and Moving Averages in Nosocomial Infection Cluster Detection. Emerg Infect Dis 2002; 8: 1426-32.

11. Hacek DM, Suriano T, Noskin GA,Kruszynski JA, Reisberg BE, Peterson LR. Medical and economic benefit of a comprehensive infection control program that includes routine determination of microbial clonality. Am J Clin Pathol 1999; 111: 647-54.

12. Ngo L Tager IB, Hadley D. Application of exponential smoothing for nosocomial infection surveillance. Am J Epidemiol 1996; 143: 637-47.

13. Farrington CP, Andrews NJ, Beale AD, Catchpole MA. A statistical algorithm for the early detection of outbreaks of infectious disease. Journal of the Royal Statistical Society Series A 1996; 159: 547-63.

14. Hoeck MRI et al. Bacterial pathogens of hospital infections with special resistances and multiresistances. Part II. Recording and Evaluation according to $\S 23$ PIA in a regional network [German]. Bundesgesundheitsbl - Gesundheitsforsch - Gesundheitsschutz 2004; 47: 363-8.

\section{Correspondence to:}

Dr. Ulrich Sagel

Institut für Mikrobiologie und Infektionsepidemiologie

Krankenanstalten Gilead/Mara

Remterweg 2

33617 Bielefeld

Germany

E-mail: mikrobiologie@gilead.de 